Research Paper

\title{
Capn4 overexpression indicates poor prognosis of ovarian cancer patients
}

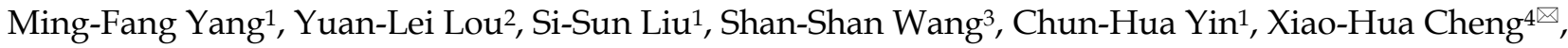 \\ Ou-Ping Huang ${ }^{\square 凶}$ \\ 1. Department of Obstetrics and Gynecology, the First Affiliated Hospital of Nanchang University, Nanchang 330006, China; \\ 2. Institute of Urology, Nanchang University, the First Affiliated Hospital of Nanchang University, Nanchang 330006, China: \\ 3. Department of Pathology, the First Affiliated Hospital of Nanchang University, Nanchang 330006, China; \\ 4. Department of Pharmacy, the First Affiliated Hospital of Nanchang University, Nanchang 330006, China; \\ 5. Department of Obstetrics and Gynecology, Jiangxi Maternal and Child Health Hospital, Nanchang 330006, China. \\ $凶$ Corresponding authors: Tel: +86-791-8869-9340, E-mail: xww@ncu.edu.cn (X-H Cheng); Tel: +86-791-8869-5051, E-mail: ncujg2009@163.com (O.-P.Huang) \\ (c) Ivyspring International Publisher. This is an open access article distributed under the terms of the Creative Commons Attribution (CC BY-NC) license \\ (https:// creativecommons.org/licenses/by-nc/4.0/). See http://ivyspring.com/terms for full terms and conditions.
}

Received: 2017.07.19; Accepted: 2017.10.24; Published: 2018.01.01

\begin{abstract}
Recent studies have shown a close correlation between Capn4 expression and the prognosis of patients with solid tumors. This study aimed to investigate clinical role of Capn4 in ovarian cancer. The expression of Capn 4 in 113 ovarian cancer and 35 non-tumor tissue samples were detected by quantitative real-time polymerase chain reaction (qRT-PCR). Capn4 expression was significantly upregulated in ovarian cancer tissues compared with non-tumor tissues $(P<0.01)$, and was positively correlated to FIGO stage, tumor grade and distant metastasis of ovarian cancer. Kaplan-Meier analysis indicated that patients with high Capn4 expression had shorter overall survival $(\mathrm{HR}=1.929,95 \% \mathrm{Cl}: 1.210-3.077, \mathrm{P}=0.006)$ and progress-free survival $(\mathrm{PFS})(\mathrm{HR}=2.043$, 95\%Cl: 1.276-3.271, $\mathrm{P}=0.003)$. Moreover, univariate Cox regression analysis demonstrated that Capn4 overexpression was an unfavorable prognostic factor for ovarian cancer $(\mathrm{HR}=2.819,95 \% \mathrm{Cl}$ : 1.365-3.645, $\mathrm{P}=0.003$ ). After the adjustment with age, histological type and tumor size, multivariate Cox regression analysis showed that Capn4 expression level $(\mathrm{HR}=2.157,95 \% \mathrm{Cl}: 1.091-3.138, \mathrm{P}=$ $0.014)$, distant metastasis $(\mathrm{HR}=1.576,95 \% \mathrm{Cl}$ : $1.025-3.012, \mathrm{P}=0.028)$, tumor grade $(\mathrm{HR}=1.408$, $95 \% \mathrm{Cl}: 0.687-2.884, \mathrm{P}=0.037)$, and FIGO stage $(\mathrm{HR}=1.791,95 \% \mathrm{Cl}: 1.016-3.158, \mathrm{P}=0.036)$ were independent poor prognostic indicators for ovarian cancer. In conclusion, Capn4 has the potential as a new prognostic marker for patients with ovarian cancer.
\end{abstract}

Key words: ovarian cancer; Capn4; prognosis; biomarker.

\section{Introduction}

Among gynecological malignancies, ovarian cancer is the leading cause of cancer-associated death worldwide. An estimated 238,700 new cases and 151,900 deaths occurred in 2012, and 58\% of new cases occurred in developing countries [1]. Approximately 22,280 women were newly diagnosed with ovarian cancer and 14,240 women were expected to die from ovarian cancer in the United States in 2016 [2]. Recent advances in the diagnosis and treatment of ovarian cancer have led to high expectation for long-term survival of patients with early-stage disease. However, a limited improvement in survival rates has been achieved over the past decade. Ovarian cancer remains extremely difficult to treat, and the 5-year survival rate remains below $40 \%$ [3]. Like many other types of malignancy, ovarian cancer progresses through processes that include initiation, local progression and metastasis, which are likely associated with a wide variety of genetic aberrations $[4,5]$. Furthermore, most ovarian cancer patients are diagnosed at an advanced stage characterized by extensive invasion and metastasis, and these patients have an extremely poor prognosis and a high mortality rate. However, the precise mechanisms of 
ovarian cancer invasion and metastasis remain undefined. Therefore, it is necessary to identify new prognostic biomarkers and therapeutic targets to improve treatment strategies.

Calpain is a highly conserved family of calcium-dependent, non-lysosomal cysteine proteases that selectively catalyze the proteolysis of many specific substrates involved in various cell processes, including motility, proliferation and apoptosis [6-10]. Thus far, fifteen calpain isoforms have been identified and characterized in mammals [11]. Among them, calpain-1 and calpain-2, which contain large $80-\mathrm{kDa}$ catalytic subunits, are ubiquitously expressed and are primarily considered cytoplasmic enzymes. Capn4 is a small regulatory subunit $(28 \mathrm{kDa})$ of the calpain proteolytic system and plays an essential role in maintaining calpain-1 and calpain-2 stability and activity [12]. Accumulating evidence has indicated that defects in Capn4 lead to the dysfunction of calpain-1 and calpain-2, which can in turn cause early embryonic lethality [13]. Recent studies have shown a close correlation between Capn 4 expression and the prognosis of patients with solid tumors, such as glioma [14], hepatocellular carcinoma [15], nasopharyngeal carcinoma [16] and breast cancer [17]. Furthermore, siRNA-mediated silencing of Capn4 resulted in markedly decreased migration, invasion and proliferation of cancer cells [18-20].

However, it is still unclear whether Capn 4 could serve as a potential biomarker or as a novel therapeutic target for the early diagnosis and treatment of ovarian cancer. Thus, in this study we examined Capn4 expression in ovarian cancer tissues using IHC, qRT-PCR and Western blot analyses. In addition, correlation analysis was performed to investigate the association between Capn 4 expression and clinicopathological features and prognosis in ovarian cancer patients.

\section{Materials and Methods}

\section{Patients}

A total of 113 ovarian cancer and 35 non-tumor tissue samples were obtained from patients undergoing surgical resection in the Department of Obstetrics and Gynecology at the First Affiliated Hospital of Nanchang University (Nanchang, China) between January 2006 and December 2010. Ovarian cancer and non-tumor tissue specimens were collected from the patients, immediately frozen in liquid nitrogen after removal and maintained at $-80^{\circ} \mathrm{C}$ until use. None of the enrolled patients received chemotherapy or radiotherapy prior to surgery. Diagnosis and differentiation of ovarian cancer were defined according to World Health Organization criteria [21]. According to postoperative pathology, 98 patients received six cycles of platinum-based chemotherapy as follows: paclitaxel $\left(135 \mathrm{mg} / \mathrm{m}^{2}\right)$ and cisplatin $\left(75 \mathrm{mg} / \mathrm{m}^{2}\right)$ or paclitaxel $(175 \mathrm{mg} / \mathrm{m} 2)$ and carboplatin (AUC6). This study was approved by the Ethics Committee of the first Affiliated Hospital of Nanchang University (Ethical Approval No. 2013023; Date:12 March 2013), and informed consent was obtained from each patient. The main clinical and pathological features, including age, histological subtype, FIGO stage, tumor size, distant metastasis and tumor grade, are summarized in Table 1. All the patients got follow-up for 60 months from the date of surgical resection. Overall survival (OS) was defined as the period of time between the date of the initial surgery and death or the last follow-up. Progression-free survival (PFS) was defined as the date from the initial surgery to the date of disease progression or the date of patient death from any cause.

Table 1. Correlations between Capn4 expression and clinicopathological parameters in ovarian cancer patients

\begin{tabular}{|c|c|c|c|c|c|c|}
\hline \multirow[t]{2}{*}{ Parameter } & \multirow[t]{2}{*}{ Group } & \multirow[t]{2}{*}{ Total } & \multicolumn{2}{|c|}{ Number of patients } & \multirow[t]{2}{*}{$p$-Value } & \multirow[t]{2}{*}{$\mathrm{X}^{2}$} \\
\hline & & & High & Low & & \\
\hline \multirow[t]{3}{*}{ Age (years) } & & & & & 0.054 & 3.703 \\
\hline & $<55$ & 51 & $22(43.14 \%)$ & $29(56.86 \%)$ & & \\
\hline & $\geq 55$ & 62 & $38(61.29 \%)$ & $24(38.71)$ & & \\
\hline \multirow{6}{*}{$\begin{array}{l}\text { Histological } \\
\text { type }\end{array}$} & & & & & 0.226 & 5.658 \\
\hline & serous & 69 & $33(47.83 \%)$ & $36(52.17 \%)$ & & \\
\hline & mucinous & 8 & $4(50.00 \%)$ & $4(50.00 \%)$ & & \\
\hline & endometrioid & 11 & $8(72.73 \%)$ & $3(27.27 \%)$ & & \\
\hline & clear-cell & 10 & $4(40.00 \%)$ & $6(60.00 \%)$ & & \\
\hline & mixed & 15 & $11(73.33 \%)$ & $4(26.67 \%)$ & & \\
\hline \multirow[t]{3}{*}{ FIGO stage } & & & & & $0.004^{\star *}$ & 8.143 \\
\hline & $\mathrm{III}+\mathrm{IV}$ & 73 & $46(63.01 \%)$ & $27(36.99 \%)$ & & \\
\hline & $\mathrm{I}+\mathrm{II}$ & 40 & $14(35.00 \%)$ & $26(65.00 \%)$ & & \\
\hline \multirow{3}{*}{$\begin{array}{l}\text { Tumor size } \\
(\mathrm{cm})\end{array}$} & & & & & 0.508 & 0.437 \\
\hline & $<10$ & 76 & $42(55.26 \%)$ & $34(44.74 \%)$ & & \\
\hline & $\geq 10$ & 37 & $18(48.65 \%)$ & $19(51.35 \%)$ & & \\
\hline \multirow{3}{*}{$\begin{array}{l}\text { Distant } \\
\text { metastasis }\end{array}$} & & & & & $0.014^{*}$ & 5.995 \\
\hline & Yes & 50 & $33(66.00 \%)$ & $17(34 \%)$ & & \\
\hline & No & 63 & $27(42.86 \%)$ & $36(57.14 \%)$ & & \\
\hline \multirow[t]{3}{*}{ Tumor grade } & & & & & $0.014^{*}$ & 6.051 \\
\hline & G1 & 44 & $17(38.64 \%)$ & $27(61.36 \%)$ & & \\
\hline & $\mathrm{G} 2+\mathrm{G} 3$ & 69 & $43(62.32 \%)$ & $26(37.68 \%)$ & & \\
\hline
\end{tabular}

$* \mathrm{P}<0.05, * * \mathrm{P}<0.01$

\section{Immunohistochemistry (IHC)}

Paraffin-embedded ovarian cancer tissues were cut into $5-\mu \mathrm{m}$ sections and then subjected to IHC using standard protocols. The primary antibody for Capn4 (Santa Cruz Biotechnology, Santa Cruz, CA, USA) was diluted 1:100. To avoid bias, the slides were independently reviewed by two experienced pathologists blinded to the clinical data. The 
percentage and intensity of Capn4-positive cells in randomly selected fields were assessed to determine Capn4 expression as described previously [22]. Briefly, the percentage was scored as follows: $0(0 \%), 1$ $(0 \%-10 \%), 2(10 \%-50 \%)$, and $3(>50 \%)$. The intensity was scored as follows: 0 (negative staining), 1 (weak staining), 2 (moderate staining), and 3 (intense staining). The final scores of Capn4 expression, ranging from 0 to 9 , were calculated by multiplying the percentage score by the intensity score. Patients with a final score of $<4$ were classified as low expression group and vice versa.

\section{qRT-PCR}

Total RNA was extracted from ovarian cancer tissues and non-tumor tissues using TRIzol (Invitrogen, Carlsbad, CA, USA). First-strand cDNA was synthesized with a Prime Script Reverse Transcriptase Kit (TaKaRa, Dalian, China) according to the manufacturer's instructions. qRT-PCR was performed on a CFX96 Real-Time PCR Detection System (Bio-Rad, Hercules, CA, USA). The specific primers were as follows: GAPDH forward 5'-TCCACCACCCTGTTGTGTA-3', and reverse 5'-ACCACAGTCCATGCCATCAC-3'; Capn4 forward 5'-TCCGACGCTACTCAGATGAAAGT-3', and reverse 5'-GATTTGTCCAGTGCCATCTTTGT-3'. Relative changes in Capn 4 mRNA expression were normalized to GAPDH and calculated using the $2^{-\Delta \Delta \mathrm{Ct}}$ method. All experiments were performed in triplicate.

\section{Western blot analysis}

Protein was extracted from frozen ovarian cancer tissues and non-tumor tissues using a Whole Protein Extraction Kit (Fermentas Life Science, Glen Burnie, $\mathrm{MD}$, USA) according to the manufacturer's instructions. Western blot analysis was performed as described previously [23]. A polyclonal rabbit anti-human antibody against Capn4 (1:1000; Abcam, Cambridge, MA, USA) and a goat anti-rabbit immunoglobulin G antibody conjugated with horseradish peroxidase (1:5000; Invitrogen) were used. GAPDH (1:5000; Abcam, Cambridge, MA, USA) was used as loading control. All experiments were performed in triplicate.

\section{Statistical Analysis}

Statistical analysis was performed using SPSS statistical software version 20.0 (SPSS Inc., Chicago, IL, USA) for Windows. Data were expressed as the mean \pm standard deviation (SD). Student's t-test was used to compare quantitative data between groups. Categorical data were analyzed using $\mathrm{X}^{2}$ test or Fisher's exact test. The cumulative progression-free survival (PFS) and OS rates were calculated using the Kaplan-Meier method, and differences were compared using a log-rank test. A Cox proportional hazards regression model was used for univariate and multivariate analyses. $\mathrm{P}<0.05$ was considered statistically significant.

\section{Results}

\section{Capn4 gene expression is abnormally increased in ovarian cancer tissues}

We determined the protein and mRNA expression levels of Capn 4 in 113 ovarian tumor and 35 non-tumor tissues. Western blot analysis revealed that Capn 4 protein levels were significantly increased in ovarian cancer tissues compared with non-tumor tissues ( $\mathrm{P}<0.01$, Fig. 1 A). qRT-PCR showed that Capn4 mRNA levels were significantly higher in ovarian cancer tissues than non-tumor tissues $(\mathrm{P}<$ 0.01, Fig. 1 B). Furthermore, IHC analysis showed positive immunoreactivity for Capn 4 primarily in the cytoplasm of ovarian cancer tissues (Fig. 1 C).

\section{Capn4 expression is positively associated with clinicopathological features in ovarian cancer patients}

To evaluate the significance of Capn4 expression in ovarian cancer, we examined the associations between Capn4 expression and several clinicopathological features in 113 patients with ovarian cancer. As shown in Table 1, high Capn4 expression showed no association with patient age $(\mathrm{P}$ $=0.054)$, histological subtype $(\mathrm{P}=0.226)$ or tumor size $(\mathrm{P}=0.508)$. However, high Capn4 expression was positively correlated with FIGO stage $(P=0.004)$, tumor grade $(\mathrm{P}=0.014)$ and distant metastasis $(\mathrm{P}=$ 0.014). Taken together, these results support that Capn 4 promotes the progression and development of ovarian cancer.

\section{High Capn4 expression is correlated with poor prognosis in ovarian cancer patients}

To further assess the potential clinical value of Capn4 expression for the prognosis in ovarian cancer patients, Kaplan-Meier survival curve analysis and log-rank tests were performed to predict OS and PFS in 113 ovarian cancer patients. The results showed that high expression of Capn4 was negatively correlated with OS (HR=1.929, 95\%CI: 1.210-3.077, $p=$ $0.006)$ and PFS (HR $=2.043,95 \% \mathrm{CI}: 1.276-3.271, \mathrm{p}=$ 0.003) $(\mathrm{P}<0.01$, Fig. 2).

Univariate Cox proportional hazards regression model analysis showed that Capn4 expression level $(\mathrm{HR}=2.819,95 \% \mathrm{CI}: 1.365-3.645, \mathrm{P}=0.003)$, distant metastasis $(\mathrm{HR}=2.013,95 \% \mathrm{CI}: 1.253-3.422, \mathrm{P}=0.004)$, tumor grade $(\mathrm{HR}=1.825,95 \% \mathrm{CI}$ : 0.806-3.279, $\mathrm{P}=$ 0.025), and FIGO stage (HR = 2.141, 95\%CI:1.286-3.563, P = 0.006) were correlated with OS 
rate in ovarian cancer patients $(\mathrm{P}<0.05$, Table 2$)$. In a multivariate Cox proportional hazards model, Capn4 expression level $(\mathrm{HR}=2.157,95 \% \mathrm{CI}: 1.091-3.138, \mathrm{P}=$ $0.014)$, distant metastasis $(\mathrm{HR}=1.576,95 \% \mathrm{CI}$ : $1.025-3.012, \mathrm{P}=0.028)$, tumor grade $(\mathrm{HR}=1.408$,
95\%CI: 0.687-2.884, $\mathrm{P}=0.037)$, and FIGO stage $(\mathrm{HR}=$ 1.791, 95\%CI: $1.016-3.158, \mathrm{P}=0.036)$ were found to be independent prognostic indicators for OS $(\mathrm{P}<0.05$, Table 2). These data suggest that Capn4 is a potential prognostic biomarker for ovarian cancer.

A

$\begin{array}{llllllll}\mathrm{N} 1 & \mathrm{~T} 1 & \mathrm{~N} 2 & \mathrm{~T} 2 & \mathrm{~N} 3 & \mathrm{~T} 3 & \mathrm{~N} 4 & \mathrm{~T} 4\end{array}$

B
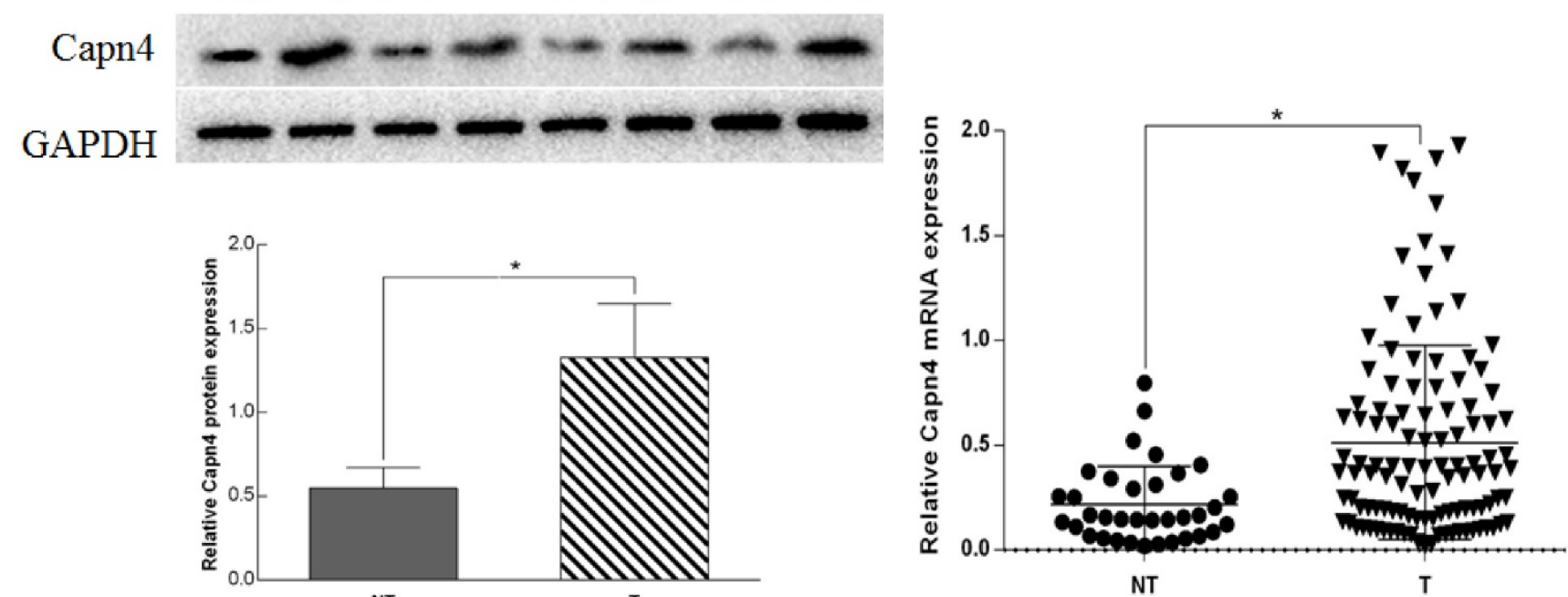

C
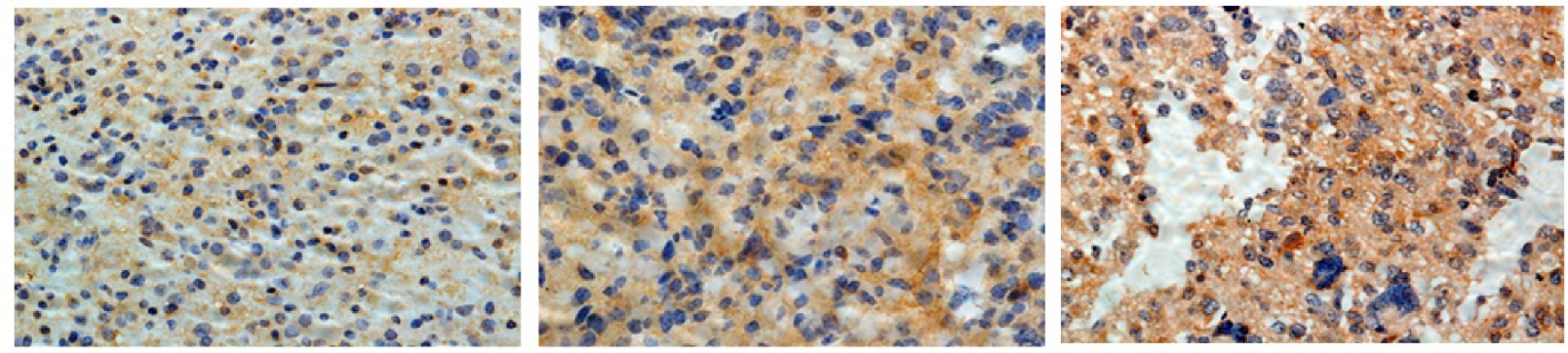

Figure 1. Relative expression of Capn4 in ovarian cancer tissues and non-tumor tissues. (A) Capn4 protein expression levels in adjacent non-tumor tissues (NT) and tumor tissues (T) from four patients assessed by Western blot analysis. ${ }^{*} \mathrm{P}<0.01$. (B) $q$ RT-PCR analysis of Capn4 expression levels in ovarian cancer tissues and adjacent non-tumor tissues $(n=24)$. $* P<0.01$. (C) IHC staining of Capn4 expression in ovarian cancer tissues (left panel: low expression; middle panel: moderate expression; right panel: high expression). Magnification fold: $x 400$.

A

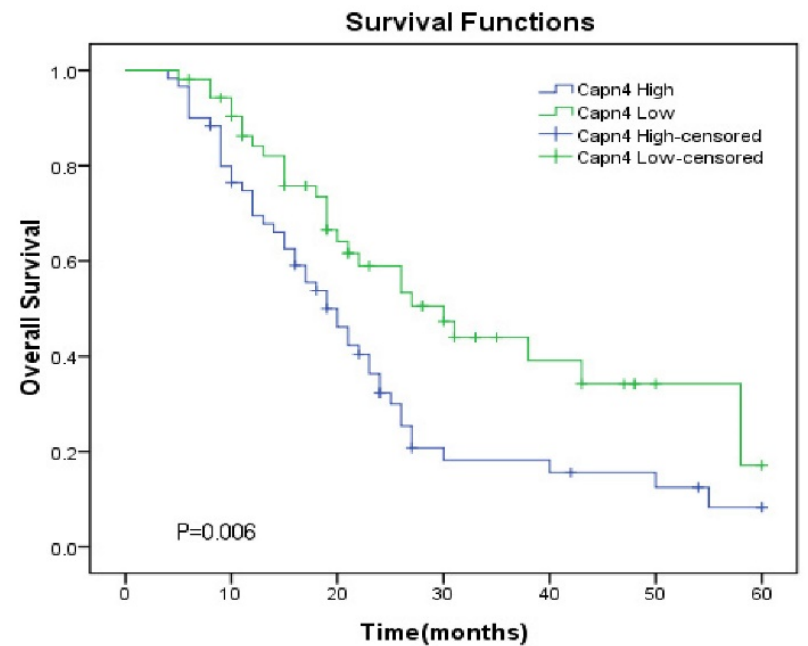

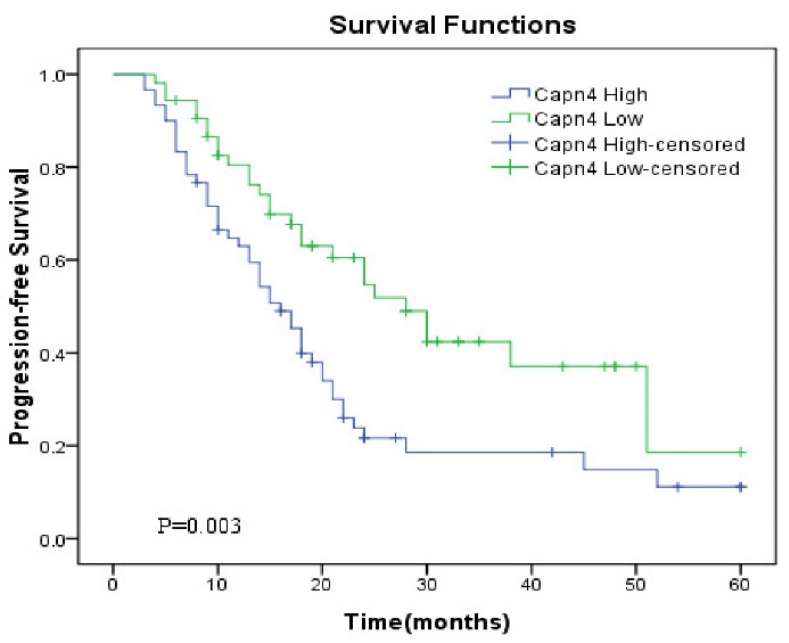

Figure 2. Kaplan-Meier survival curves for patients with ovarian cancer according to Capn4 expression level. (A) Overall survival (OS) and (B) progression-free survival (PFS) curves. Patients with high Capn4 expression levels exhibited a poor prognosis with respect to OS $(P=0.006)$ and $P F S(P=0.003)$. 
Table 2. Univariate and multivariate Cox regression analyses of prognostic factors in ovarian cancer patients

\begin{tabular}{|c|c|c|c|c|}
\hline \multirow[t]{2}{*}{ Variable } & \multicolumn{2}{|l|}{ Univariate analysis } & \multicolumn{2}{|l|}{ Multivariate analysis } \\
\hline & $\mathrm{HR}(95 \% \mathrm{CI})$ & $p$-Value & $\mathrm{HR}(95 \% \mathrm{CI})$ & $p$-Value \\
\hline Age (years) (<55 vs. >55) & $0.903(0.539-1.660)$ & 0.824 & & \\
\hline $\begin{array}{l}\text { Histological type (serous vs. } \\
\text { mucinous+endometrioid+clear-cell+mixed) }\end{array}$ & $1.169(0.709-1.928)$ & 0.541 & & \\
\hline FIGO stage (I+II vs. III+IV) & $2.141(1.286-3.563)$ & $0.006^{* *}$ & $1.791(1.016-3.158)$ & $0.036^{*}$ \\
\hline Tumor size $(\mathrm{cm})(<10$ vs. $\geq 10)$ & $1.325(0.758-3.056)$ & 0.188 & & \\
\hline Distant metastasis (yes vs. no) & $2.013(1.253-3.422)$ & $0.004^{* *}$ & $1.576(1.025-3.012)$ & $0.028^{*}$ \\
\hline Tumor grade (G1 vs. G2+G3) & $1.825(0.806-3.279)$ & $0.025^{*}$ & $1.408(0.687-2.884)$ & $0.037^{*}$ \\
\hline Capn4 expression level (high vs. low) & $2.819(1.365-3.645)$ & $0.003^{* *}$ & $2.157(1.091-3.138)$ & $0.014^{*}$ \\
\hline
\end{tabular}

$* \mathrm{P}<0.05, * * \mathrm{P}<0.01$

\section{Discussion}

Ovarian cancer is the most common and lethal malignancy in women. Most women are diagnosed with advanced-stage disease, characterized by extensive invasion and metastasis as well as poor prognosis. Up to now, no sensitive and specific biomarkers have been identified for early diagnosis of ovarian cancer.

The small regulatory subunit Capn 4 plays an essential role in maintaining calpain stability and activity, and calpains play important role in cytoskeletal remodeling, proteolytic modification, gene expression regulation and substrate degradation [24]. Aberrant expression of Capn4 is prevalent in a wide spectrum of cancers and high levels of Capn4 are associated with poor patient outcomes [15-18]. Therefore, Capn 4 might be a candidate diagnosis biomarker and a therapy target for cancer. However, there has no report on Capn4 expression levels in ovarian cancer.

The current study aimed to detect the expression levels of Capn4 in ovarian cancer tissues. To our knowledge, this is the first report to show that the expression levels of Capn 4 are dramatically elevated in ovarian cancer tissues compared with non-tumor tissues, consistent with the high expression of Capn4 in glioma and hepatocellular carcinoma [14, 15]. In addition, we explored the association between Capn 4 expression and the clinicopathological features of patients with ovarian cancer. Interestingly, patients with high Capn4 expression levels exhibited parameters associated with poor prognosis, including FIGO stage, tumor grade, and distant metastasis, suggesting that Capn4 expression plays a crucial role in the progression of ovarian cancer. In particular, increased Capn4 expression was correlated with an increased tendency toward malignancy. Therefore, we conclude that high levels of Capn4 are correlated with ovarian cancer progression.

High Capn4 expression has been strongly linked to advanced clinical stage and poor prognosis in a variety of solid cancers, and it was demonstrated to be an especially accurate prognostic indicator in patients with non-small cell lung cancer or intrahepatic cholangiocarcinoma $[25,26]$. Therefore, we utilized Kaplan-Meier survival analysis to examine the correlation between Capn4 expression and the survival outcomes of ovarian cancer patients. The results showed that patients with high Capn4 expression had notably decreased OS and PFS compared with those with low Capn4 expression. Moreover, multivariate Cox regression analysis indicated that Capn4 expression could serve as a powerful independent predictor of the prognosis for patients with ovarian cancer, consistent with previous findings that high Capn4 expression promoted tumor development and predicted poor prognosis $[14,16,25$, 26].

However, this study has limitations because we did not explore the precise mechanism by which Capn4 affects prognosis and outcome in ovarian cancer patient. Further experiments in cell and animal models of ovarian cancer are needed to understand biological processes regulated by Capn 4 in ovarian cancer progression.

In conclusion, we report that Capn4 is highly expressed in ovarian cancer tissues and its expression is positively correlated with FIGO stage, tumor grade and distant metastasis of ovarian cancer. Moreover, ovarian cancer patients with high capn4 expression had poor prognosis. These findings suggest that Capn 4 could serve as a novel prognostic biomarker for ovarian cancer.

\section{Acknowledgments}

This study was supported by the National Nature Science Foundation of China (No. 81260381).

\section{Competing Interests}

The authors have declared that no competing interest exists.

\section{References}

1. Torre LA, Bray F, Siegel RL, Ferlay J, Lortet-Tieulent J, Jemal A. Global Cancer Statistics, 2012. CA Cancer J Clin. 2015;65(2):87-108.

2. Siegel RL, Miller KD, Jemal A. Cancer statistics, 2016. CA Cancer J Clin. 2016;66(1):7-30. 
3. Colombo PE, Fabbro M, Theillet C, Bibeau F, Rouanet P, Ray-Coquard I. Sensitivity and resistance to treatment in the primary management of epithelial ovarian cancer. Crit Rev Oncol Hematol. 2014;89(2):207-216.

4. Eismann S, Vetter L, Keller M, et al. Long-term experiences with genetic consultation in people with hereditary breast and ovarian cancer. Arch Gynecol Obstet. 2016;294(5):1011-1018.

5. Huang S, Qing C, Huang Z, Zhu Y. The long non-coding RNA CCAT2 is up-regulated in ovarian cancer and associated with poor prognosis. Diagn Pathol. 2016;11(1):49.

6. Goll DE, Thompson VF, Li H, Wei W, Cong J. The calpain system. Physiol Rev.2003; 83(3):731-801

7. Franco SJ, Huttenlocher A. Regulating cell migration: calpains make the cut. J Cell Sci. 2005;118(Pt 17):3829-3838.

8. Glading A, Lauffenburger DA, Wells A. Cutting to the chase: calpain proteases in cell motility. Trends Cell Biol. 2002;12(1):46-54.

9. Bhatt A, Kaverina I, Otey C, Huttenlocher A. Regulation of focal complex composition and disassembly by the calcium-dependent protease calpain. I Cell Sci. 2002;115(Pt 17):3415-3425.

10. Tan Y, Wu C, De Veyra T, Greer PA. Ubiquitous calpains promote both apoptosis and survival signals in response to different cell death stimuli. J Biol Chem. 2006;281(26):17689-17698.

11. Saez ME, Ramirez-Lorca R, Moron FJ, Ruiz A. The therapeutic potential of the calpain family: new aspects. Drug Discov Today. 2006;11(19-20):917-923.

12. Arthur JS, Elce JS, Hegadorn C, Williams K, Greer PA. Disruption of the murine calpain small subunit gene, Capn4: calpain is essential for embryonic development but not for cell growth and division. Mol Cell Biol. 2000;20(12):4474-4481.

13. Dourdin N, Bhatt AK, Dutt P, et al. Reduced cell migration and disruption of the actin cytoskeleton in calpain-deficient embryonic fibroblasts. J Biol Chem. 2001;276(51):48382-48388

14. Cai JJ, Qi ZX, Hua W, et al. Increased expression of Capn4 is associated with the malignancy of human glioma. CNS Neurosci Ther. 2014;20(6):521-527.

15. Dai Z, Zhou SL, Zhou ZJ, et al. Capn4 contributes to tumor growth and metastasis of hepatocellular carcinoma by activation of the FAK-Src signalling pathways. J Pathol. 2014;234(3):316-328.

16. Zheng PC, Chen X, Zhu HW, et al. Capn4 is a marker of poor clinical outcomes and promotes nasopharyngeal carcinoma metastasis via nuclear factor-kB-induced matrix metalloproteinase 2 expressions. Cancer Sci. 2014;105(6):630-638

17. Wang GS, Huang YG, Li H, Bi SJ, Zhao JL. ERK/CANP rapid signaling mediates $17 \beta$-estradiol-induced proliferation of human breast cancer cell line MCF-7 cells. Int J Clin Exp Med. 2014;7(1):156-162

18. Zhang F, Wang Q, Ye L, Feng Y, Zhang X. Hepatitis B virus X protein upregulates expression of calpain small subunit-1 via nuclear factor-kappaB/p65 in hepatoma cells. J Med Virol. 2010;82(6):920-928.

19. Li Y, Zhang Z, Zhou X, et al. The oncoprotein HBXIP enhances migration of breast cancer cells through increasing filopodia formation involving MEKK2/ERK1/2/Capn4 signaling. Cancer Lett. 2014;355(2):288-296.

20. Dourdin N, Bhatt AK, Dutt P, et al. Reduced cell migration and disruption of the actin cytoskeleton in calpain-deficient embryonic fibroblasts. J Biol Chem. 2001;276(51):48382-48388

21. Kurman RJ, Carcangiu ML, Herrington CS, Young RH. WHO Classification of Tumours of Female Reproductive Organs. Fourth Edition. 2014.

22. Gao H, Yao Q, Lan X, et al. Elevated HABP1 protein expression correlates with progression and poor survival in patients with gastric cancer. Onco Targets Ther. 2016;9:6711-6718.

23. Li Z, Zhang G, Li D, et al. Methylation-associated silencing of miR-495 inhibit the migration and invasion of human gastric cancer cells by directly targeting PRL-3. Biochem Biophys Res Commun. 2015;456(1):344-350.

24. Storr SJ, Carragher NO, Frame MC, Parr T, Martin SG. The calpain system and cancer. Nat Rev Cancer. 2011;11(5):364-374.

25. Gu J, Xu FK, Zhao GY, et al. Capn 4 promotes non-small cell lung cancer progression via upregulation of matrix metalloproteinase 2. Med Oncol. 2015;32(3):51.

26. Zhang C, Bai DS, Huang XY, et al. Prognostic significance of Capn4 overexpression in intrahepatic cholangiocarcinoma. PLoS One. 2013;8(1):e54619. 